Revue Française de Civilisation Britannique

XVII-2 | 2012

Minorités, intégration en Grande-Bretagne et dans les pays du Commonwealth

\title{
Le post-multiculturalisme de David Cameron
}

David Cameron's Post-Multiculturalism

Didier Lassalle

\section{(2) OpenEdition}

1 Journals

Édition électronique

URL : http://journals.openedition.org/rfcb/688

DOI : $10.4000 / \mathrm{rfcb} .688$

ISSN : 2429-4373

Éditeur

CRECIB - Centre de recherche et d'études en civilisation britannique

Édition imprimée

Date de publication : 15 octobre 2012

Pagination : 191-212

ISBN : 2-911580-37-0

ISSN : 0248-9015

Référence électronique

Didier Lassalle, «Le post-multiculturalisme de David Cameron », Revue Française de Civilisation

Britannique [En ligne], XVII-2 | 2012, mis en ligne le 15 mars 2016, consulté le 10 décembre 2020. URL :

http://journals.openedition.org/rfcb/688 ; DOl : https://doi.org/10.4000/rfcb.688

Ce document a été généré automatiquement le 10 décembre 2020.

\section{cc) (†)}

Revue française de civilisation britannique est mis à disposition selon les termes de la licence Creative Commons Attribution - Pas d'Utilisation Commerciale - Pas de Modification 4.0 International. 


\title{
Le post-multiculturalisme de David Cameron
}

\author{
David Cameron's Post-Multiculturalism
}

Didier Lassalle

1 À Munich, lors d'une récente conférence internationale consacrée à la sécurité, David Cameron s'est violemment attaqué au «multiculturalisme d'état » (state multiculturalism) à la britannique. Il a ainsi accusé cette doctrine d'être à l'origine du séparatisme communautaire et du climat d'aliénation qui constituent, selon lui, le compost sur lequel prospère le terrorisme des fondamentalistes musulmans. Il a appelé de ses vœux un « libéralisme musclé » (muscular liberalism) capable à la fois de faire face à l'extrémisme islamiste et de promouvoir une identité britannique inclusive ${ }^{1}$. Son discours, calibré à la perfection, a eu les effets escomptés en déclenchant, au plan national, une véritable tempête médiatique : les Conservateurs se félicitant bruyamment de la fin annoncée du multiculturalisme ; le porte-parole de l'opposition travailliste pour la justice, Sadiq Khan, accusant le Premier ministre de faire le jeu de l'extrême droite; les groupes musulmans et antifascistes mettant en cause ses intentions en faisant remarquer que son discours coïncidait étrangement avec une manifestation organisée le même jour par le parti d'extrême droite English Defence League (EDL) à Luton² .

2 Pourtant, l'argumentaire développé par David Cameron à Munich n'était pas nouveau puisqu'il l'avait déjà éprouvé lors d'un débat organisé par la Commission pour l'Égalité et les Droits Humains (Equality and Human Rights Commission) en 2008. Il affirmait à l'époque que : «le multiculturalisme - l'idée que différentes cultures doivent être respectées au point de les encourager à vivre séparément - a dangereusement sapé le sentiment identitaire de la Grande-Bretagne et entrainé un «apartheid culturel « ${ }^{3}$. Il ajoutait qu'il était du devoir des Conservateurs de s'assurer que tous les immigrants apprennent l'anglais, de promouvoir le droit des femmes et de lutter contre l'extension du séparatisme dans le pays ${ }^{4}$.

D'autre part, le multiculturalisme avait déjà subi de sérieuses attaques bien avant son arrivée au pouvoir en mai 2010. En 2002, les prises de position de David Blunkett, alors 
ministre de l'Intérieur, sur l'impératif linguistique et la nécessité d'un minimum d'intégration culturelle de la part des minorités allaient déjà dans ce sens ${ }^{5}$. Elles faisaient suite aux deux rapports analysant les causes ainsi que le déroulement des émeutes de l'été 2001 à Bradford, Burnley et Oldham qui avaient dénoncé les effets négatifs de la «balkanisation ethnique» sur la cohésion sociale dans ces villes ${ }^{6}$. Dans un discours prononcé en 2005, le président de la CRE, Trevor Phillips, stigmatisait déjà les dérives d'un multiculturalisme «à l'américaine » qui conduisait tout droit vers un renforcement de la ségrégation et des inégalités ${ }^{7}$. En 2006, Ruth Kelly, ministre de l'éducation, se réjouissait même de l'effritement rapide du consensus multiculturaliste accusé d'avoir favorisé la séparation entre les communautés ${ }^{8}$. De même, dans son discours Our Nation's Future multiculturalism and integration de décembre 2006, Tony Blair insistait tout particulièrement sur le "devoir d'intégration" (the duty to integrate) des minorités ethniques compatible, selon lui, avec leur droit à la différence. Il n'hésitait pas non plus à dénoncer les dérives de la « diversité » qui conduisaient à la séparation des communautés et au rejet des valeurs fondamentales de la société britannique ${ }^{9}$. Les controverses sur le port du voile islamique, déclenchées par Jack Straw (alors président des Communes), ainsi que les inquiétudes exprimées par Trevor Phillips sur l'éventualité d'émeutes à la française dans les quartiers sensibles soulignaient que le revirement idéologique était déjà bien engagé $^{10}$. De même, Gordon Brown, dans son discours de 2008 (Managed Migration and Earned Citizenship), souhaitait que les nouveaux arrivants fassent preuve d'un plus grand désir de rejoindre le "projet collectif» que représente la nation britannique ${ }^{11}$. Il reprenait à son compte le concept très controversé de "citoyenneté méritée » (earned citizenship) développé par David Goodhart le rédacteur en chef de Prospect Magazine ${ }^{12}$.

Enfin, lorsqu'il était encore dans l'opposition, David Cameron dénonçait avec verve la dangereuse confusion introduite, selon lui, par le gouvernement New Labour entre cohésion sociétale, intégration des musulmans britanniques et menace terroriste. Son insistance à découpler les questions d'intégration et de cohésion des problèmes sécuritaires lui avait même valu les louanges du Muslim Council of Great-Britain (MCB) ${ }^{13}$. Le discours de Munich revient partiellement sur cette position. En effet, il y insiste sur les conséquences néfastes du séparatisme communautaire - encouragé par des leaders charismatiques qualifiés d' " extrémistes non-violents »- qui conduirait les musulmans à se définir uniquement en fonction de leur appartenance religieuse. Par un processus de contagion, l'enfermement au sein de ce maillage serré de croyances radicales amènerait les plus influençables ou les plus déterminés à basculer dans la violence terroriste ${ }^{14}$.

5 En surface, il existe donc une filiation évidente entre la politique développée par les gouvernements New Labour en matière d'intégration à partir de 2001 et celle désormais impulsée par le gouvernement de coalition de David Cameron. Plus en profondeur, des différences notables apparaissent tant sur la forme que sur le fond. Mais avant de les discuter en détail, il est nécessaire de se pencher sur le contexte sociopolitique britannique qui permet de mieux appréhender la politique actuelle en matière d'intégration et de cohésion sociétale. Trois éléments majeurs sont à considérer : les craintes suscitées dans la population britannique par le volume croissant du solde migratoire ; la montée en puissance de l'islamophobie et la multiplication des réactions xénophobes; la prise en compte du 'white backlash' engendré par la mise en place des politiques multiculturelles. 


\section{Immigration : I'habituel bouc-émissaire}

6 Depuis le discours enflammé d'Enoch Powell de 1968 dénonçant l'immigration en provenance du Nouveau Commonwealth et critiquant la mise en place d'une législation antidiscriminatoire, la question de l'immigration est récurrente dans le débat politique au Royaume-Uni ${ }^{15}$. Examinons d'abord la réalité des estimations chiffrées fournies par l'ONS sur la période 1997-2009 qui couvre pratiquement l'ensemble de la gestion New Labour. Le tableau $n^{\circ} 1$ indique un solde migratoire global de plus de 2,2 millions de personnes dont environ la moitié (1,1 million) est issue des pays du Nouveau Commonwealth. Ces arrivées massives ont largement contribué à l'accroissement général de la population britannique qui est passée de 58,3 à 61,8 millions sur la même période ${ }^{16}$.

Tableau $\mathrm{n}^{\circ} 1$ : Long-Term International Migration. Time series 1991 to 2009 by Country of last or next residence, UK - balance inflow/outflow (thousands).

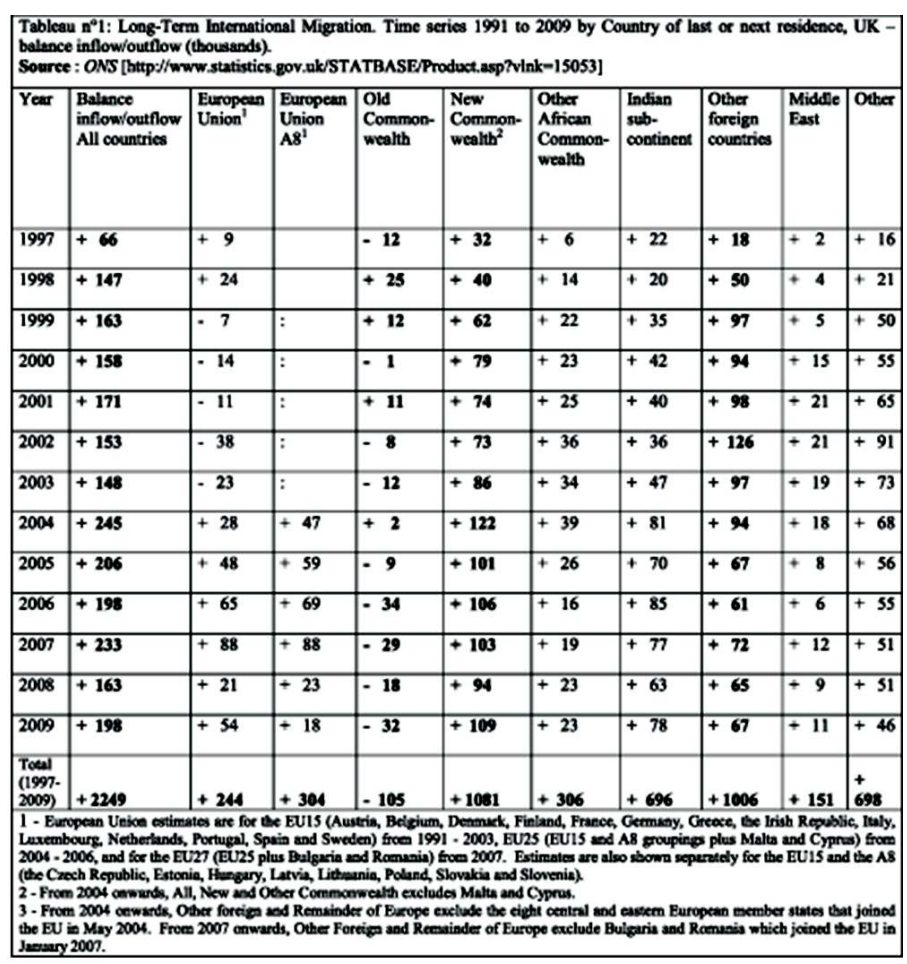

source : ONS [http://www.statistics.gov.uk/STATBASE/Product.asp?vlnk=15053]

7 À ces chiffres, il convient d'ajouter un nombre relativement important de migrants en situation irrégulière, dont l'estimation peut varier du simple au double selon les sources. Une étude publiée en 2009 propose le chiffre moyen de 618000 personnes pour l'année 2007 sur l'ensemble du pays - compris dans une fourchette qui va de 417000 pour l'estimation basse à 863000 pour la plus haute ${ }^{17}$. La conclusion manifeste est que la gestion d'ensemble du phénomène migratoire par les trois gouvernements New Labour s'est traduite par l'augmentation massive de la population étrangère résidant au Royaume-Uni : environ 3 millions de personnes supplémentaires.

8 Le contrôle de l'immigration devient une question politique centrale pour le New Labour dans les années 2000. Dans un premier temps, le second gouvernement Blair assouplit la 
règlementation afin de répondre aux besoins du pays en personnel qualifié, comme il s'y était engagé dans son programme électoral de $2001^{18}$. Les Conservateurs accusent alors les Travaillistes de sous-estimer le problème et ses conséquences néfastes pour l'avenir du pays ainsi que d'avoir abandonné leurs engagements de $1997^{19}$. En 2003, un groupe de pression très marqué à droite, MigrationWatch UK, se constitue et lance une campagne d'opinion dans la presse (Daily Mail, Daily Telegraph, Daily Express, Daily Star mais également le Times et le Guardian) contre le supposé laxisme gouvernemental en matière d'immigration et de droit d'asile. Son credo est celui de la migration équilibrée (balanced migration) qui consiste à rapprocher au maximum les volumes des flux migratoires entrant et sortant ${ }^{20}$. Ses conceptions radicales en matière de contrôle de l'immigration seront ensuite relayées à la Chambre des Communes par l'intermédiaire d'un groupe «trans-partis » (Cross-Party Group on Balanced Migration) dirigé par les députés Frank Field (travailliste) et Nicholas Soames (conservateur) en 2008.

9 À partir de 2004, l'économie britannique, qui joue à fond le jeu de la mondialisation, emploie une main d'œuvre étrangère abondante et bon marché provenant, en particulier, des pays nouvellement entrés dans l'Europe (A8 countries) au détriment de la maind'œuvre locale qui se retrouve au chômage et commence à protester sérieusement ${ }^{21}$. Dans un discours de 2007, le Premier ministre, Gordon Brown, reprend alors la rhétorique habituellement associée au BNP: il déclare vouloir créer des "emplois britanniques » pour les "travailleurs britanniques »". La même année, le Select Committee on Economic Affairs de la Chambre des Lords publie un rapport concernant la politique migratoire du gouvernement dans lequel l'impact de l'immigration sur l'économie et la société britannique est analysé. Ses conclusions sont sans appel: « we have found no evidence for the argument, made by the Government, business and many others, that net immigration immigration minus emigration - generates significant economic benefits for the existing UK population " ${ }^{23}$. En 2008, les premiers effets de la crise des "subprimes» se font sentir avec pour corollaire une dégradation continue des chiffres de l'emploi, qui conduit à des manifestations et des grèves sauvages dans de nombreuses raffineries et centrales électriques du pays début 2009. À cette occasion, les travailleurs expriment leurs inquiétudes concernant l'avenir compromis de leurs emplois et reprennent à leur compte la phrase malheureuse du Premier ministre ${ }^{24}$. Quelques semaines plus tard, le ministre en charge de l'immigration, Phil Woolas, réutilise sans complexe le même argumentaire protectionniste lorsqu'il insiste sur la nécessité de réformer le système de permis de travail à point (points-based system) afin de restreindre le nombre des immigrants qualifiés (hors UE) et de permettre ainsi à plus de diplômés britanniques de décrocher un emploi ${ }^{25}$.

10 Plus gênant, en 2009, un ancien conseiller et rédacteur de discours de Tony Blair, Andrew Neather, dévoile dans un article de presse la politique concertée d'ouverture des frontières des premiers gouvernements travaillistes destinée, selon lui, à transformer radicalement le pays en le rendant véritablement multiculturel ${ }^{26}$. Ses affirmations, discutables, sont reprises avec délectation par des chroniqueurs de droite, comme Mélanie Phillips, qui étrillent les gouvernements travaillistes successifs, coupables à leurs yeux d'avoir favorisé la montée du BNP en négligeant les aspirations et les intérêts de leurs propres électeurs issus de la classe ouvrière ${ }^{27}$. À partir de cette date, le virage ultraprotectionniste amorcé par le gouvernement de Gordon Brown en 2007 en matière d'immigration est totalement assumé. Par exemple, la ministre de l'Intérieur Jacqui Smith déclare aux journalistes de la $B B C$ que les immigrants ne devraient pas pouvoir occuper un emploi qualifié au Royaume-Uni qui n'ait été préalablement proposé aux travailleurs 
britanniques ; son successeur Alan Johnson reconnait pour sa part la «maladresse » des administrations Conservatrice et New Labour précédentes et le fait que certaines régions du pays ont été particulièrement affectées par l'immigration qui a pesé lourdement sur le marché de l'emploi et les services publics locaux ${ }^{28}$. Le rapport du Select Committee on Economic Affairs de la Chambre des Lords, mentionné précédemment, leur donne en partie raison, puisque tout en soulignant le faible nombre des études réalisées sur ce sujet, il indique :

The available evidence suggests that immigration has had a small negative impact on the lowest-paid workers in the UK, and a small positive impact on the earnings of higher-paid workers. Resident workers whose wages have been adversely affected by immigration are likely to include a significant proportion of previous immigrants and workers from ethnic minority groups ${ }^{29}$.

11 Les partis extrémistes surfent sur l'hostilité croissante à l'égard des immigrants. Le British National Party (BNP) demande l'arrêt immédiat de toute immigration (pays de l'Union Européenne inclus) ainsi que la mise en place d'un programme de rapatriement volontaire qui coïncide étrangement avec la réhabilitation d'Enoch Powell et de ses idées. Les nationalistes de l'UKIP, quant à eux, exigent le gel pour cinq ans de toute immigration permanente et l'instauration de permis de travail pour les citoyens européens. Enfin, lors de la campagne électorale de 2010, Gordon Brown, dans l'espoir de regagner les faveurs d'un électorat populaire très hostile à l'immigration, n'hésite pas à fustiger les «immigrants illégaux»tout en appelant cyniquement les trois grands partis à condamner la xénophobie ${ }^{30}$.

Ainsi adoubée par les plus hauts représentants de l'État, l'idée que les étrangers immigrés menacent les moyens d'existence d'une partie non négligeable de la population britannique (les travailleurs les moins qualifiés) s'ancre de plus en plus fermement dans l'opinion. Une enquête réalisée par Ipsos Mori en 2007 indique que 77 \% des Britanniques interrogés s'inquiètent des conséquences possibles de l'immigration sur le fonctionnement des services publics de santé et d'éducation dans leur localité, et $82 \%$ des conséquences possibles au plan national. De plus, ils sont $80 \%$ à ne pas faire confiance au gouvernement quant à l'ampleur réelle de l'immigration ${ }^{31}$. L'enquête comparative, Transatlantic Trends: Immigration, réalisée en 2010 souligne que l'immigration est le problème politique majeur pour $23 \%$ des Britanniques et que, d'autre part, ces derniers sont les plus réservés des Européens sur l'apport de l'immigration, avec $65 \%$ des sondés considérant qu'elle constitue plutôt un problème qu'une chance pour le pays ${ }^{32}$. Ce sentiment est largement répandu dans toutes les strates de la société. Par exemple, l'enquête de l'institut Populus met en évidence une convergence de vue manifeste au sein des différentes communautés qui constituent la société britannique. En effet, $34 \%$ des Blancs et $39 \%$ des Asiatiques sont favorables à une ligne générale très restrictive concernant la politique d'immigration. Les deux raisons principales invoquées étant d'une part, que les immigrants constituent une menace pour l'emploi pour $40 \%$ des Asiatiques et $23 \%$ des Blancs, et d'autre part, qu'ils ont un effet négatif sur le niveau des salaires pour $47 \%$ des Asiatiques et $34 \%$ des Blancs ${ }^{33}$. Enfin, l'amplification de la crise économique qui secoue le pays et fait monter en flèche le nombre des chômeurs contribue à renforcer dans l'opinion ces perceptions négatives de l'immigration et des migrants. Les dernières enquêtes confirment cette impopularité croissante. Celle du Citizenship Survey 2010 montre que $78 \%$ des personnes interrogées sont favorables à une réduction de l'immigration, dont $56 \%$ favorables à une forte diminution ${ }^{34}$. 


\section{Islamophobie et xénophobie : fruits amers du double discours sécuritaire et protectionniste}

13 Les émeutes du printemps et de l'été 2001 (qui ont constitué un sévère avertissement pour ceux qui pensaient que l'intégration était sur la bonne voie), les attentats du 11 septembre, l'intervention militaire en Afghanistan et la reprise du conflit avec l'Irak, ont réactivé le sentiment de méfiance à l'égard des musulmans vivant sur le territoire britannique. Les attentats meurtriers de Londres en juillet 2005, triste illustration du fossé qui s'est creusé entre certains jeunes musulmans et la société britannique dans son ensemble, l'ont radicalisé. De plus, la montée concomitante des revendications religieuses, culturelles et éducatives de ces communautés, souvent associée à l'apparition de formes contemporaines de fondamentalisme religieux, sont suivies avec hostilité par la plupart des politiques et des acteurs sociaux. Cet amalgame de craintes infondées entourant la foi islamique et ses règles de vie, s'exprime dans les médias par l'intermédiaire de stéréotypes négatifs, parfois très violents, visant les pays musulmans et la culture musulmane en général ainsi que les communautés britanniques de cette obédience religieuse, qui sociologiquement regroupent les personnes les plus touchées par la pauvreté et le chômage, tels les Pakistanais, les Bangladais ou les Somaliens. La presse populaire fustige leur intransigeance, leur esprit antidémocratique et le caractère fondamentaliste de leurs croyances et de leurs pratiques religieuses d'autant plus que la force montante de l'Islam, même modéré, inquiète énormément ${ }^{35}$. Ainsi, la chroniqueuse Yasmin Alibhai-Brown n'hésite pas à dénoncer le courant raciste et xénophobe qui se répand dans la société britannique, où ce type de discours est désormais considéré comme acceptable puisqu'il se contente de stigmatiser la présence de ces "'étrangers' immigrés [qui] sont un désastre pour la nation». Elle critique aussi férocement les intellectuels et les médias qui, selon elle, ont contribué à le rendre respectable ${ }^{36}$. La conséquence la plus néfaste de ces dérives est que le discours radical des petits partis d'extrême droite ouvertement islamophobe, tels que le National Front, le BNP ou le mouvement plus récent mais très actif de l'English Defence League (créé en 2009 à Luton), sort progressivement de sa marginalité et bénéficie de ces circonstances favorables pour diffuser largement dans l'opinion.

Pour sa part, Iqbal Sacranie, ancien secrétaire général du Muslim Council of Britain (MCB), déclare dans une interview réalisée en avril 2011 :

The last few months have seen the curse of Islamophobia on the increase; we have seen a number of nasty and unpleasant incidents happening in the community including mosques being vandalised, desecration of Muslim graves, women being attacked because of wearing Hijab or Nikab and list goes on. With all such attacks happening, the mainstream media is either silent or ignorant at worst of these antiMuslim hate crimes. What one finds amazing instead of showing signs of concern to good community relations and social cohesion by reporting these incidents, the right wing media continues to pursue its agenda of denigrating Islamic values by concentrating on the un-Islamic acts of the few which have been condemned by mainstream Muslim organisations ${ }^{37}$.

15 La baronne Warsi (co-présidente du parti Conservateur et ministre sans portefeuille du gouvernement de David Cameron) quant à elle, met en garde contre la banalisation de l'islamophobie qui serait désormais considérée comme «normale et acceptable lors des dîners $»^{38}$. Cette affirmation est confortée par le sondage commandé par la fondation 
Searchlight Educational Trust à l'institut Populus en 2010 qui montre que l'Islam et les musulmans font l'objet de jugements extrêmement négatifs dans de nombreux secteurs de la population : plus de la moitié des personnes interrogées pensent que les musulmans «create problems in the UK » et $43 \%$ soutiendraient même une campagne destinée à empêcher la construction d'une mosquée dans leur quartier ${ }^{39}$.

Nous avons vu précédemment que la stigmatisation s'est progressivement étendue à l'ensemble des immigrés au cours de la décennie 2000. En effet, des argumentations à caractère nationaliste et xénophobe font leur apparition pour condamner l'arrivée massive des européens de l'Est, en particulier des Polonais, accusés de faire baisser les salaires et de prendre les emplois des travailleurs britanniques ${ }^{40}$. Ceci conduit le gouvernement New Labour à abandonner sa politique de la "porte-ouverte » (" open-door " policy) en imposant des restrictions à la mobilité des travailleurs roumains et bulgares, pays nouvellement entrés dans l'UE, en janvier 2007. Il s'agit de ne pas reproduire la même erreur qu'avec les pays du groupe A8 et de limiter l'influx prévisible de ces nouveaux citoyens, qui déstabiliserait encore un peu plus le marché de l'emploi. Cette mesure marque un tournant de la politique migratoire du gouvernement qui, dans le discours politique du moins, devient plus restrictive et prend un caractère véritablement protectionniste. Curieusement, la fameuse déclaration de Gordon Brown («British jobs for British workers ») fut très critiquée à l'époque par l'opposition Conservatrice conduite par David Cameron qui dénonça son illégalité au regard de la législation européenne ainsi que le recyclage politicien de l'argumentaire raciste du BNP au cours d'un vif débat parlementaire ${ }^{41}$.

\section{Multiculturalisme et « white backlash »}

L'expression «white backlash", popularisée par le livre de Roger Hewitt (2005), fait référence à la réponse des classes populaires blanches aux politiques d'égalité des droits, d'égalité des chances et antiracistes progressivement mises en place en faveur des groupes ethniques minoritaires. Dans un contexte où la politique de classe est progressivement supplantée par celle de l'identité et de la différence, certains Blancs se sentent dépossédés et développent le sentiment d'être traités d'une façon "injuste ». Ils avancent même l'argument que c'est leur propre culture, et non plus celles des minorités, qui fait l'objet d'un manque de reconnaissance de la part des autorités administratives et politiques du pays ${ }^{42}$. Depuis les années 1980, le « white backlash » et les discours associés de marginalisation matérielle et culturelle - engendrés par la croyance que les avantages dont bénéficiait la population blanche auraient été rognés par l'avancée du multiculturalisme - ont été récupérés par des acteurs politiques variés. Enoch Powell est le premier à avoir tenté d'instrumentaliser ces craintes à la fin des années 1960s. Les gouvernements successifs de Margaret Thatcher ont également joué cette partition avec beaucoup d'habileté, réussissant à marginaliser l'extrême droite dans le même mouvement : rejet d'une gauche jugée trop libérale et censée mettre en péril la nation britannique par sa promotion irréfléchie des politiques multiculturelles.

Depuis 1999, la presse de droite ainsi que certains hommes politiques ont réactivé ces discours dans un contexte de déclin de la souveraineté nationale, de menace terroriste, d'immigration massive, et de questionnement identitaire. C'est la publication du rapport Macpherson sur le meurtre du jeune Stephen Lawrence qui, en dénonçant le racisme institutionnel des forces de police, et plus généralement des institutions, semble en être 
le déclencheur. En effet, les critiques acerbes de Macpherson ont été interprétées dans la presse de droite comme une attaque contre l'« Anglicité » ("Englishness») ainsi qu'une capitulation en ras-campagne de l'élite cosmopolite dirigeante « anti-anglaise » devant le concept étranger du " politiquement correct $»^{43}$. L'idée est alors avancée que si le racisme est le résultat de la non-prise en compte institutionnelle des spécificités de chaque groupe, alors les Anglais constituent le groupe le plus mal loti puisqu'ils ne peuvent prétendre à aucun privilège ${ }^{44}$. Dans le même temps, en dépit d'un positionnement relativement conservateur des gouvernements New Labour sur les questions de la diversité et du multiculturalisme, ces derniers ont été vilipendés pour leur incapacité à contrôler les frontières, pour avoir imposé la dictature du politiquement correct et pour avoir échoué à réduire les inégalités sociales persistantes qui transcendent les frontières de la 'race' et de l'appartenance ethnique.

Bien entendu, le BNP s'est engouffré dans cette brèche en se présentant comme le champion de la population blanche laissée pour compte et appelant à la «justice " pour les Blancs («Rights for Whites») dans des zones sensibles telles que Burnley ou Barking et Dagenham. C'est au cœur des bastions traditionnels ouvriers du parti travailliste que le $B N P$ a recueilli le plus de transfuges, mais il a également attiré des militants provenant des deux autres grands partis politiques et issus de toutes les strates de la classe ouvrière et de la basse classe moyenne ${ }^{45}$. De plus, des personnalités politiques de premier plan ont tenté d'utiliser ces dangereux leviers pour s'assurer un gain politique ou ne pas perdre leurs électeurs. Pendant la campagne électorale de 2005, le chef du parti Conservateur, Michael Howard, a combiné une ligne politique très dure sur l'immigration et une critique cinglante du « politiquement correct ${ }^{46}$. Les Travaillistes ne sont pas en reste. En 2003, le député d'Oldham East et de Saddleworth, Phil Woolas, critique la police qui, selon lui, ne traite pas les attaques racistes contre les Blancs de la même manière que celles ciblant les personnes issues des minorités ethniques ${ }^{47}$. De même, la députée de Barking, Margaret Hodge, a exprimé son inquiétude de voir les Blancs négligés par l'ensemble des grands partis à de nombreuses reprises. En 2006, elle fait sensation en déclarant que les familles de la classe ouvrière blanche se sentent tellement abandonnées par le gouvernement et qu'elles sont si mécontentes du niveau élevé de l'immigration qu'elles désertent le parti pour aller directement s'inscrire au BNP :

They can't get a home for their children, they see black and ethnic minority communities moving in and they are angry [...]. When I knock on doors I say to people, 'are you tempted to vote BNP?' and many, many, many - eight out of 10 of the white families - say 'yes'. That's something we have never seen before, in all my years. Even when people voted BNP, they used to be ashamed to vote BNP. Now they are not ${ }^{48}$.

En 2009, la ministre en charge des Communautés (Communities Secretary), Hazel Blears, admet que les Blancs de la classe ouvrière pensent parfois que personne ne les écoute ou ne parle en leur faveur et qu'ils devraient être autorisés à exprimer leurs inquiétudes légitimes sans craindre de se faire traiter de racistes ${ }^{49}$. Cela fait suite à un rapport publié par ses services qui souligne que cette population se sent « trahie » et « abandonnée » par le personnel politique des grands partis qui les place généralement en seconde position, derrière les immigrants, dans ses préoccupations. Tel est l'un des points clés de ce rapport:

Where immigration and integration are discussed in depth as problematic, there is a focus on real or perceived competition for resources; housing, benefits, jobs, territory and national culture. The implications of this for the political capital that 
can be accrued by the Far-right are very grave. Our white interviewees' responses to minorities are far from universally negative. In fact everything from indifference, through empathy, a desire for more and better engagement, to anxiety was registered in these interviews. People express a desire for equality and a level playing field, not only in economic terms, but also in terms of ethnic groups (and even sections of ethnic groups). In this reading, there is injustice and unfairness because the same rules do not seem to apply to everyone. However, the assumptions about who is entitled to resources seem to lean toward a racial base, with local variations ${ }^{50}$.

21 Le député travailliste Frank Field, co-président du groupe "trans-parti » pour une « immigration équilibrée », en profite pour insister sur le danger politique que constitue à ses yeux l'inaction gouvernementale dans ce domaine :

No wonder people feel the government is riding roughshod over their wishes, and not only in the poorest areas, which are bearing the brunt of the present massive level of immigration. Unless further action is taken soon, immigration will add nearly 10 million to the population of England in the next 20 years. [...] If Labour wants to influence the outcome of the next general election, it had better start addressing white working-class concern about immigration, not simply reporting on it ${ }^{51}$.

\section{Ébauche d'une politique post-multiculturelle spécifique ou simple reconditionnement des politiques du New Labour?}

Lors de la campagne électorale de 2010, David Cameron s'était fermement engagé sur trois points afin de rendre plus efficaces les politiques d'intégration et de contrôle de l'immigration qui, selon lui, n'avaient pas été suffisamment prises au sérieux par les gouvernements précédents. En premier lieu, et pour répondre aux souhaits de son électorat ainsi que d'une partie non-négligeable de l'électorat travailliste qu'il comptait séduire, il avait proposé de limiter l'immigration drastiquement en introduisant une limite supérieure annuelle fixée par le gouvernement en fonction des besoins. En second lieu, il avait promis d'en finir avec les errements 'désastreux' du multiculturalisme, qu'il n'avait d'ailleurs jamais cessé de critiquer depuis son accession à la direction du parti Conservateur. Enfin, il prônait un retour à une vision assimilationniste classique de ce qu'il appelait l'« intégration » ainsi qu'un renforcement de la « cohésion sociétale ».

Dès son arrivée au pouvoir, le gouvernement de coalition se fixe l'objectif de réduire le solde annuel du flux migratoire. La ministre de l'Intérieur, Theresa May, confirme la mise en place de restrictions immédiates limitant l'admission des travailleurs hors UE et souligne la volonté gouvernementale: «What we have as an aim is indeed to bring immigration down from the hundreds of thousands that it became under Labour to the tens of thousands that it used to $b e^{52}$ ». Cependant, cette ligne politique ferme, très en phase avec les attentes d'une large majorité de la population, fait aussitôt l'objet de sérieuses critiques. La focalisation $d u$ gouvernement sur la réduction du solde migratoire en est la première cible car elle souligne son impuissance à réellement contrôler le phénomène: le solde migratoire dépend autant de l'immigration que de l'émigration et le gouvernement n'a aucun moyen d'influencer cette dernière. D'autre part, le contrôle de l'immigration est limité par les traités internationaux, ce qui réduit d'autant sa marge de manœuvre. Par exemple, il est désormais impossible d'empêcher l'arrivée de travailleurs polonais, bulgares ou roumains 
dans le pays car ils ont légalement le droit de s'y installer en tant que citoyens de l'UE, ce qui provoque l'ire et renforce l'argumentaire d'un parti nationaliste et anti-européen comme le United Kingdom Independence Party (UKIP). De même, la Convention internationale sur le droit d'asile, la Convention Européenne des Droits de l'Homme (CEDH), la Charte des Droits Fondamentaux de l'Union Européenne, constituent des contraintes importantes, en particulier pour ce qui concerne la migration familiale. La décision récente d'étendre les exigences en termes de compétence linguistique aux conjoints de Britanniques souhaitant s'installer dans le pays pourrait ainsi faire l'objet d'un recours sur la base de l'article 8 de la $\mathrm{CEDH}^{53}$. Enfin, la volonté gouvernementale de réduire l'immigration en provenance des pays hors zone UE se heurte aux besoins en main d'œuvre qualifiée des entreprises, et celle de faire la chasse aux "faux étudiants » ("bogus students») aux intérêts économiques des universités et des nombreuses écoles de langue du pays ${ }^{54}$. Le gouvernement a cédé en exemptant de toute restriction les transferts de personnels au sein des grands groupes industriels (intracompany transfers (ICTs)) qui représentaient plus de la moitié des visas d'entrée délivrés en $2010: 29175$ sur un total de 55 935. Cette exemption, largement exploitée par les grands groupes, constitue une faille importante dans le dispositif comme l'indiquent les statistiques puisque seulement 22030 ICTs avaient été accordés l'année précédente ${ }^{55}$.

Lors d'un discours de campagne pour les élections locales de 2011, le Premier ministre a dénoncé le rôle ambigu joué par le précédent gouvernement dans le débat sur l'immigration. Selon lui, les Travaillistes auraient tenté de le museler en accusant leurs adversaires de racisme tout en validant dans leurs déclarations les arguments de l'extrême-droite :

The last government, in contrast, actually helped to inflame the debate. On the one hand, there were Labour ministers who closed down discussion, giving the impression that concerns about immigration were somehow racist. On the other, there were ministers hell bent on burnishing their hardline credentials by talking tough but doing nothing to bring the numbers down. [...]It created the space for extremist parties to flourish, as they could tell people that mainstream politicians weren't listening to their concerns or doing anything about them ${ }^{56}$.

Pourtant, au cours de la même intervention, il défendait lui-même des positions susceptibles de populariser un certain type de discours xénophobe. Tout en reconnaissant certains bénéfices à l'immigration, il soulignait que l'immigration massive de ces dernières années était responsable des tensions et de l'inconfort ressentis dans certains quartiers en raison de la pression supplémentaire qu'exerçaient les nouveaux arrivants sur les services sociaux de la santé, du logement et sur les établissements scolaires publics. Il stigmatisait aussi les immigrés incapables de parler l'anglais ainsi que ceux qui ne voulaient pas ou n'étaient pas vraiment disposés à s'intégrer. Les nationalistes de l' UKIP n'ont pas manqué de relever une certaine convergence avec leurs idées. Nigel Farage, le dirigeant de cette formation politique, s'est réjoui que David Cameron reconnaisse enfin le caractère néfaste de l'immigration de masse mais a déclaré aussitôt que le pays ne retrouverait sa pleine liberté en matière de gestion des flux migratoire qu'une fois hors de l'UE. Pour sa part, le BNP l'a accusé d'opportunisme cynique pour avoir pillé leurs idées sur le sujet quelques semaines avant ces échéances électorales ${ }^{57}$. Les attaques sont également venues de son propre gouvernement. Vince Cable, le ministre du Commerce et de l'Industrie, et ancien vice-président du Parti libéral-démocrate a déclaré dans la presse que les propos du Premier ministre sur l'immigration étaient «très imprudents », et Nick Clegg, le vice-Premier ministre a fait savoir qu'il en avait pris 
connaissance sans les approuver. Le chef du Parti travailliste, Ed Miliband, est alors en mesure d'ironiser à son tour sur les contradictions qui se font jour au sein de la coalition gouvernementale: "it's hard to have a government policy that is clear and coherent if your business secretary, who's in charge of your student visa policy, is saying one thing, and actually going out of his way to attack the prime minister ${ }^{58} \%$.

Bien que le gouvernement conservateur ne puisse pas encore en être tenu pour responsable, les récentes statistiques publiées par l'ONS montrent combien il est difficile de faire des prévisions fiables dans ce domaine: le solde migratoire sur la période comprise entre septembre 2009 et septembre 2010 a augmenté de $80 \%$ par rapport à la période précédente (passant de 147000 à 243000 ). Ce chiffre résulte de la combinaison de deux facteurs inattendus: une baisse du nombre des émigrants; une reprise de l'immigration en provenance des pays de l'Est de l'UE. Pour Matt Cavanagh, le directeur adjoint de l'Institute of Public Policy Research (IPPR), les Conservateurs n'ont pas tenu compte de la baisse possible de l'émigration lorsqu'ils ont fixé leur objectif de réduction du solde migratoire. Comme il est très délicat de réduire sensiblement l'immigration familiale, ils seront contraints de limiter fortement le nombre des étudiants et des travailleurs qualifiés hors UE (qui constituent leur seule réelle variable d'ajustement) pour l'atteindre, ce qui pénalisera l'économie britannique car cette immigration est la plus intéressante de ce point de vue ${ }^{59}$.

Le Premier ministre, qui risque sa crédibilité politique sur cette question, est aussi continuellement aiguillonné par le lobby anti-immigration très actif. Ce dernier a marqué des points en obtenant pour la première fois un débat à la Chambre des Communes sur ce sujet délicat grâce au dépôt d'une motion. Le texte de cette dernière, présentée par Frank Field, était sans la moindre équivoque :

That this House calls on Her Majesty's Government to act on the overwhelming public concern about the present scale of immigration by taking firm measures to reduce immigration without excluding those individuals who are genuinely essential to economic recovery, on which so much else depends.

Deux arguments principaux ont été avancés. Le premier est typiquement malthusien : il est absolument vital de contrôler le nombre des immigrants afin de limiter l'accroissement considérable prévu de la population britannique (70 millions en 2030 80 millions en 2050) ce qui constituerait un fardeau économique et social bien trop lourd pour le pays, surtout en termes d'éducation, de santé et de logement. Le second est plus pragmatique et prétend favoriser l'intégration : les immigrés doivent absolument parler l'anglais pour participer pleinement à la vie sociale et démocratique de leur pays d'accueil 60. De même, l'ancien archevêque de Canterbury et membre du groupe "trans-parti » pour une immigration équilibrée (Cross-Party Group on Balanced Migration), George Carey, a affirmé que la masse des immigrants en provenance d'Europe et d'ailleurs plaçait non seulement les ressources du pays sous une énorme pression mais menaçait l'ADN même de la nation ${ }^{61}$.

La doctrine multiculturaliste et le muticulturalisme d'État ont constitué deux cibles privilégiées pour les attaques politiques de David Cameron lorsqu'il était chef de l'opposition conservatrice. Dans un discours de 2007, il en pointait déjà les failles, responsables selon lui de l'affaiblissement du sentiment de cohésion nationale. Il dénonçait en particulier l'accent placé sur ce qui divise plutôt que sur ce qui rassemble la nation : la sanctuarisation du «droit à la différence » (un concept clivant) au détriment $\mathrm{du}$ «droit à un traitement égal» en dépit des différences (un concept unificateur). Il 
proposait en outre quelques pistes : s'assurer que tous les citoyens britanniques soient capables de s'exprimer dans la langue nationale afin qu'ils puissent communiquer entre eux ; renforcer l'enseignement de l'histoire britannique dans les écoles et multiplier les occasions de célébrer le sentiment d'identité nationale ${ }^{62}$. Dans son intervention de 2010 à Munich en qualité de Premier ministre, il reprend une partie de ces arguments tout en accusant le multiculturalisme d'État d'avoir encouragé «different cultures to live separate lives, apart from each other and apart from the main stream " et d'avoir instauré une politique de complaisance passive irresponsable qui a longtemps toléré que "these segregated communities behave in ways that run completely counter to our values " à condition que ces communautés respectent les $\operatorname{lois}^{63}$. La doctrine multiculturaliste serait ainsi passée, dans une sorte de dérive épistémologique, de la tolérance des autres cultures à la tolérance d'autres systèmes de valeurs, dont certains peuvent être hostiles au pays. À la place, il propose de mette en place un « libéralisme musclé » (" muscular liberalism») qui n'hésite pas à s'opposer à toutes les formes d'extrémisme et à promouvoir activement une série de valeurs communes britanniques non négociables: liberté d'expression, liberté de culte, démocratie, l'État de droit, l'égalité des droits quels que soient l'appartenance ethnique, le sexe ou l'orientation sexuelle ${ }^{64}$. Enfin, un document récent définit plus précisément les orientations politiques de son gouvernement de coalition en matière d'égalité des droits. Il s'agit d'en finir avec le "politiquement correct », l'ingénierie sociale et la culture de consignation bureaucratique générée par la législation des "Race Relations», chers aux idéologues du New Labour. Cette nouvelle approche (Equality Strategy) est donc censée prendre ses distances avec la "politique identitaire » (« identity politics») du passé, en reconnaissant et en valorisant l'individu plutôt que son appartenance :

Equality is not an add-on, but an integral part of this government's commitment to build a stronger economy and fairer society. This strategy sets out a new approach to delivering equality: one that moves away from treating people as groups or 'equality strands' and instead recognizes that we are a nation of 62 million individuals ${ }^{65}$.

On apprend pourtant dans la section 3 de ce même document que, conformément à la logique de la "Big Society" développée par le Premier ministre lors de la dernière campagne électorale, le gouvernement reconnaît et souhaite valoriser la «contribution précieuse et significative» que les communautés religieuses, les associations communautaires locales, les institutions caritatives et les associations culturelles, apportent à la société dans son ensemble en leur donnant plus de pouvoir ${ }^{66}$. De même, le gouvernement doit reconnaître que des groupes ethniques ou religieux (Antillais, Pakistanais, Gens du voyage, Musulmans) sont victimes d'inégalités persistantes sur le marché de l'emploi, dans l'éducation et dans la santé. Pour y remédier, il conservera donc certains aspects de la politique précédente, mais sous une nouvelle appellation (Equality Duty $)^{67}$. Un multiculturalisme « allégé » en quelque sorte.

31 De nombreuses critiques se sont élevées contre le discours de Munich qui reprend beaucoup d'idées aux gouvernements précédents tout en les présentant comme novatrices. C'est ainsi que le concept de "Britishness » fait l'objet d'un extraordinaire recyclage alors qu'il était devenu, au fil du temps, une véritable obsession pour Tony Blair et Gordon Brown. En 2005, ce dernier avait même évoqué l'éventualité d'introduire une journée dédiée à la nation (British day) dans le calendrier. En 2007, deux de ses ministres, Ruth Kelly et Liam Byrne respectivement Communities Secretary et Immmigration Minister avaient réclamé un "national 'Britain' day» afin de renforcer la citoyenneté ${ }^{68}$. Il est donc particulièrement ironique qu'après avoir tourné Gordon Brown en ridicule pour sa 
campagne en faveur de la 'britannité', David Cameron utilise un cadre de référence pratiquement identique pour encourager certaines communautés à s'intégrer ${ }^{69}$. De même, les valeurs qu'il propose sont extrêmement générales et n'ont vraiment rien de spécifiquement britannique : elles pourraient théoriquement s'appliquer à l'ensemble des démocraties occidentales. Un aspect plus contesté est l'enthousiasme immodéré du Premier ministre pour les écoles confessionnelles (faith schools) qu'il souhaite voir se développer. Si un grand nombre de ces écoles confessionnelles ne sont en réalité que des écoles d'État sélectives déguisées qui répondent aux exigences des classes moyennes dans leur recherche d'homogénéité sociale et culturelle, d'autres sont nettement plus inquiétantes. Par exemple, les écoles chrétiennes évangéliques, hindouistes, juives orthodoxes ou privées musulmanes peuvent légalement consacrer près de la moitié de leurs enseignements journaliers à l'étude de la théologie ou du Coran et faire pratiquement l'impasse sur les matières artistiques et littéraires. Il y a donc danger à subventionner ces écoles non-étatiques de manière indiscriminée, ce qui reste pourtant le cas général pour les établissements confessionnels autres que musulmans ${ }^{70}$.

En dépit des précautions oratoires du Premier ministre, ce sont surtout les communautés musulmanes qui ont fait l'objet de ses attaques et de ses préoccupations en matière d'intégration et de prévention du terrorisme. Ainsi, il a réactivé le lien entre cohésion sociétale, intégration des Musulmans britanniques et menace terroriste, un amalgame auquel il s'était fermement opposé comme chef de l'opposition. Ce revirement était d'autant plus notable que le Premier ministre avait choisi d'aborder les thèmes de l'intégration, de l'immigration, du multiculturalisme et des échecs de certaines communautés ethniques à se forger une identité britannique spécifique, dans le cadre assez inapproprié de la Conférence sur la Sécurité de Munich : elle était parrainée, entre autres, par de grands groupes de l'industrie de la défense et de la sécurité comme Thales et Cassidian. Pourtant, en novembre 2010, Theresa May assurait encore que le gouvernement de coalition refuserait de fonder sa stratégie d'intégration sur la lutte antiterroriste comme l'avaient fait ses prédécesseurs :

But we will not securitise our integration strategy. The kind of society which we wish to encourage will not emerge through counter terrorism work. Under the last government 'Prevent' muddled up work on counterterrorism with the normal work that needs to be done to promote community cohesion and participation. Counterterrorism became the dominant way in which Government and some communities came to interact. That was wrong and no wonder it alienated so many ${ }^{71}$.

Pour David Cameron, les Musulmans sont directement ou indirectement responsables de la menace terroriste liée à l'islamisme radical. Il les accuse de promouvoir le séparatisme en se définissant avant tout comme musulmans plutôt que comme britanniques ou européens. Il insiste sur la relation conflictuelle entre culture musulmane et valeurs essentielles de la société britannique, en particulier en ce qui concerne la position des femmes ou les mariages forcés. L'implicite étant que les Musulmans britanniques sont entièrement responsables de leur propre marginalisation sociale, politique et économique. En résumé, il contribue à alimenter le 'racisme culturel' ambiant qui dépeint l'Islam sous les traits d'une religion incapable de s'acclimater au contexte britannique, et attise les flammes dangereuses de la stéréotypisation ethno-religieuse au lieu de les combattre. Les répercussions d'un argumentaire aussi brutal sur les relations parfois tendues entre Musulmans et non-musulmans sont imprévisibles mais potentiellement contre-productives en termes d'intégration. 

Bunglawala, l'un des porte-parole de Muslims4UK, a accusé le Premier ministre d'être condescendant, de tirer sur la mauvaise cible et de développer une argumentation totalement erronée ${ }^{73}$. Pour sa part, Mahmud Al-Rashid d'Emel Magazine a évoqué une occasion manquée de David Cameron: "He desecrated the memory of Muslims who had contributed to Britain; he singled out a largely powerless minority group that is constantly demonised; he conflated terrorism with cultural identity ${ }^{74}$ ". Certains pensent même que l'aboutissement logique de son discours pourrait être l'instauration d'une «citoyenneté conditionnelle " pour les Musulmans qui dépendrait de leur comportement religieux : les attributs complets de la citoyenneté leur étant attribués en fonction de leur conformité plus ou moins grande à un modèle de comportement validé par le gouvernement ${ }^{75}$. Enfin, l'utilisation de l'expression "muscular liberalism » a également été très mal perçue. D'une part elle implique l'imposition de mesures contraignantes destinées à favoriser l'intégration des Musulmans : tests de citoyenneté obligatoires, programmes de mixité ethnique et culturelle dans les domaines du logement et de l'environnement de travail, etc. D'autre part, elle renvoie au concept victorien de "muscular christianity» et à ses évidentes connotations impériales : la mission civilisatrice de l'homme blanc. Ce n'était pas la première bévue du Premier ministre dans ce domaine. En 2007, il avait déclaré: " inspiring as well as demanding loyalty from every citizen will require a new crusade for fairness » ${ }^{76}$. Une expression qui avait fait quelques vagues dans les milieux musulmans et avait nécessité une mise au point d'un de ses porte-parole: "Mr Cameron's reference to a "crusade" had not been intended to cause offence 77 ".

\section{Conclusion}

La politique de David Cameron en matière d'immigration et d'intégration est donc conditionnée par un contexte social tendu ainsi que par les fortes attentes de son électorat et de l'aile droite du parti Conservateur qui exigent un contrôle beaucoup plus strict et efficace des flux migratoires ainsi que l'abandon pur et simple des politiques multiculturelles. Ces dernières sont censées avoir conduit le pays dans une impasse sociale et identitaire en favorisant le séparatisme ethnoculturel, et avoir généré le terrorisme islamique en tolérant la mise en place de systèmes de valeurs antidémocratiques au sein de communautés issues de l'immigration, en particulier les Musulmans. L'idée centrale est qu'une 'intégration' réussie prend du temps et que ce long processus serait compromis par l'arrivée massive et continue de nouveaux immigrants, souvent incapables de communiquer avec la population autochtone et ne cherchant parfois même pas à s'intégrer. Ceci engendrerait un sentiment d'inconfort croissant ou une sensation de délitement communautaire dans les quartiers où s'installent ces nouvelles populations : des quartiers souvent déshérités où vivent également les Blancs les plus démunis de la classe ouvrière qui craignent cette concurrence venue d'ailleurs en 
matière de logement, de santé ou de prestations sociales. Les réponses que propose le Premier ministre sont d'une part la réduction drastique du solde migratoire, qui se chiffrerait en dizaine de milliers plutôt qu'en centaines de milliers comme sous les gouvernements précédents, et d'autre part, la mise en place d'un "libéralisme musclé » censé promouvoir les valeurs essentielles de la nation britannique et lutter avec acharnement contre les dérives sectaires pernicieuses.

Rien donc de très nouveau comme nous l'avons vu, puisqu'il reprend en les accentuant la plupart des idées lancées et des mesures déjà mises en place par ses prédécesseurs du New Labour dans ce domaine. Cependant, il est possible de dégager plusieurs inflexions spécifiques qui les prolongent et les amplifient. Tout d'abord, on assiste à une remise en cause extrêmement radicale de l'ensemble de la "politique identitaire » du passé, qui, à ses yeux, souffre du défaut rédhibitoire de reconnaître les appartenances communautaires au détriment de la valorisation de l'individu, et son remplacement par une nouvelle « stratégie pour l'égalité ». Cette dernière place donc l'individu - et non plus son appartenance ethnique et/ou religieuse - au centre du nouveau dispositif, met sur un même plan tous les types de discriminations ou de handicap sociaux, et cherche à instaurer un nouveau cadre institutionnel censé offrir à chacun le droit d'être traité équitablement ainsi qu'une 'véritable' égalité des chances (equal opportunity). Ensuite, la volonté d'imposer le système de valeurs britannique aux nouveaux arrivants afin de rétablir un minimum d'homogénéité culturelle marque un retour évident au concept classique d'assimilation, totalement abandonné au milieu des années 1960. Mais c'est surtout, bien qu'il s'en défende avec véhémence, sa stigmatisation de l'Islam et des Musulmans qui est la plus inquiétante, car elle pourrait conduire à isoler encore plus des communautés déjà fréquemment marginalisées et fragiliser d'autant leur « intégration » : une politique qui repose sur le « clivage » qu'il dénonçait si fermement lorsqu'il était dans l'opposition.

Ainsi, il existe une ambivalence perceptible dans la pensée post-multiculturaliste de David Cameron qui cherche à percer dans l'électorat populaire blanc viscéralement hostile à l'immigration et aux politiques multiculturelles sans toutefois s'aliéner les communautés ethniques installées. Par exemple, sa "stratégie pour l'égalité » répond clairement au sentiment d'abandon et à la demande de justice sociale de la classe ouvrière blanche. Par contre, il condamne les dérives communautaristes tout en reconnaissant que certains groupes ethniques et/ou religieux sont désavantagés économiquement et socialement, et que ceci doit être corrigé. Il n'hésite pas non plus à accorder la plus grande importance aux «communautés locales » qui constituent des rouages essentiels pour assurer le fonctionnement de la 'Big Society' dont il prône l'avènement. Cependant, le rôle qu'il compte leur faire jouer dans cette partition et leur définition exacte (politique, culturelle, éducative et confessionnelle, etc.) suscite toujours de nombreuses interrogations. 


\section{NOTES}

1. PM's speech at Munich Security Conference, Saturday 5 February 2011. [http:// www.number10.gov.uk/news/speeches-and-transcripts/2011/02/pms-speech-at-munichsecurity-conference-60293] (04/4/2011).

2. "David Cameron sparks fury from critics who say attack on multiculturalism has boosted English Defence League”, The Observer, Saturday 5 February 2011. [http://www.guardian.co.uk/ politics/2011/feb/05/david-cameron-speech-criticised-edl] (24/5/2011).

3. "multiculturalism - the idea that different cultures should be respected to the point of encouraging them to live separatel - has dangerously undermined Britain's sense of identity and brought about 'cultural apartheid"'. The Daily Mail, “'Sharia law will undermine 'British society' warns Cameron in attack on multiculturalism", 26 February 2008.

4. Ibid.

5. David BLUNKETT, "Integration with Diversity: Globalization and the Renewal of Democracy and Civil Society", in Phoebe Griffith and Mark Leonard, Reclaiming Britishness, London: The Foreign Policy Centre, 2002, pp. 65 à 77.

6. John DENHAM, Building Cohesive Communities: A report of the Ministerial Group on Public Order and Community Cohesion, London: Home Office, 2001 \& Ted CANTLE, Community cohesion: A report of the independent review team, London: Home Office, 2001.

7. Trevor PHILLIPS, Sleepwalking to segregation, discours prononcé le 22/09/2005 au Manchester Council for Community Relations, [http://www.cre.gov.uk].

8. Ruth KELLY, Integration and Cohesion, discours prononcé le 24/08/2006 lors de l'inauguration de la Commission sur l'Intégration et la Cohésion, [http://www.guardian.co.uk].

9. Tony BLAIR, discours du 8/12/2006. [http://www.number10.gov.uk].

10. "Warning over UK race riot danger", BBC NEWS, 22 October 2006, [http://www.bbc.co.uk] et "Muslim veil debate could start riots, warns Phillips", The Guardian, 23 October 2006.

11. Gordon BROWN, Managed Migration and Earned Citizenship, [http://www.number10.gov.uk/ Page14624] (15/02/2010).

12. David GOODHEART, Earned Citizenship, [http://www.pm.gov.uk/output/Page10554.asp] (4/16/2008).

13. "Cameron blast at crude bullying on 'British values", The Observer, Sunday 28 January 2007, [ http://www.guardian.co.uk/politics/2007/jan/28/uk.conservatives] (24/5/2011) et "No one will be left behind in a Tory Britain", The Observer, Sunday 28 January 2007, [ http:// www.guardian.co.uk/commentisfree/2007/jan/28/comment.conservatives] (24/5/2011).

14. PM's speech at Munich Security Conference, op.cit.

15. Le fameux 'Rivers of Blood' speech.

16. ONS, Table 1.2 - Population: constituent countries of the UK.. [http://www.statistics.gov.uk/ STATBASE/Product.asp? vlnk=15354].

17. Ian GORDON, Kathleen SCANLON Tony TRAVERS \& Christine WHITEHEAD, Economic impact on the London and UK economy of an earned regularisation of irregular migrants to the UK, LSE London, GLA Economics, 2009, table 1, p. 6.

18. "As our economy changes and expands, so our rules on immigration need to reflect the need to meet skills shortages", Labour election manifesto 2001.

19. "Every country must have firm control over immigration and Britain is no exception", Labour election manifesto 1997.

20. http://www.migrationwatchuk.org/. 
21. Cf. tableau $n^{\circ} 1$ et "Half of new jobs go to migrants", BBC NEWS UK, 30 October 2007. [http:// news.bbc.co.uk/2/hi/uk_news/politics /7069779.stm].

22. "[...] to create British jobs for British workers", BBC NEWS UK, 16 November 2007. [http:// news.bbc.co.uk/2/hi/uk_news /politics/7097837.stm].

23. House of Lords, Select Committee on Economic Affairs First Report - Abstract, session 2007-8. [ http://www.publications.parliament.uk/pa/ld200708/ldselect/ldeconaf/82/8202.htm].

24. "'British jobs for British workers': Wildcat strikes spread over foreign workers shipped into the UK”, Mail On Line, 31 January 2009. [http://www.dailymail.co.uk/news/article-1131708/ British-jobs-British-workers-Wildcat-strikes-spread-foreign-workers-shipped-UK.html] et "'British jobs for British workers' is the cry of our worst instincts", The Telegraph, 4 February 2009. [http://www.telegraph.co.uk/comment/ columnists/maryriddell /4516854/British-jobsfor-British-workers-is-the-cry-of-our-worst-instincts.html].

25. "Now it's British jobs for British graduates", The Independent, 4 February 2009. [http:// www.independent.co.uk/news/uk/politics/now-its-british-jobs-for-british graduates1544987.html].

26. Andrew NEATHER, "Dont' listen to the whingers - London needs immigrants", London Evening Standard, 23 October 2009. Andrew Neather a également travaillé pour Jack Straw et David Blunkett.

27. "The outrageous truth slips out: Labour cynically plotted to transform the entire make-up of Britain without telling us", Mail On Line, 28 October 2009. [http://www.dailymail.co.uk/ debate/ article-1222977/MELANIE-PHILLIPS-The-outrageous-truth-slips-Labour-cynically-plotted-

transform-entire-make-Britain-telling-us.html].

28. "Migrants face tighter work rules", BBC NEWS, 22 February 2009 et "Johnson admits migration mistakes”, BBC NEWS, 2 November 2009. [http://news.bbc.co.uk/go/pr/fr/-/2/hi/uk_news/ politics/8338276.stm].

29. House of Lords, Select Committee on Economic Affairs First Report, op.cit., §78.

30. "To those migrants who think they can get away without making a contribution; without respecting our way of life; without honouring the values that make Britain what it is - I have only one message - you are not welcome." in Brown steps up immigration battle, BBC NEWS, 31 March 2010.[http://news.bbc.co.uk/2/hi/uk_news/politics/8595973.stm].

31. Immigration Poll for the Sun, Ipsos Mori, November 2007. [http://www.ipsos-mori.com/content/ polls-07/immigration-poll.ashx].

32. Transatlantic Trends: Immigration, joint project of the German Marshall Fund of the United States, the Lynde and Harry Bradley Foundation, the Compagnia di San Paolo, and the Barrow Cadbury Trust, with additional support from the Fundación BBVA, 2010, Chart 1, p. 5.

33. Fear and HOPE, Searchlight Educational Trust, 2011. [http://www.fearandhope.org.uk /projectreport/ ]

34. Suzane COOPER, Citizenship Survey: April-September 2010, England, Attitudes to Immigration fig. 13, Department for Communities and Local Government, HMSO: London, 2011. [http:// www.communities.gov.uk/documents/statistics /pdf/1815799.pdf].

35. "Islamophobia pervades UK - report", BBC NEWS, 2 June 2004 et "Muslims in the European Union - Discrimination and Islamophobia”, EUMC, Austria: Manz Crossmedia, 2006, pp. 84-90. [ eumc.europa.eu/eumc/material/pub/muslim/Manifestations_EN.pdf].

36. "Powell's Rivers of Blood are back again", The Independent, 10 March 2008. [http:// www.independent.co.uk] (20/03/2008).

37. Interview réalisée par Mozammel Haque, Sir Iqbal Sacranie on Islamophobia and Multiculturalism, 17 April 2011. [http://muslimscanner.blogspot.com/2011/04/sir-iqbal-sacranie-onislamophobia-and.html].

38. "Muslim baroness warns the 'bigots': Dinner party Islamophobia is rife, says Warsi", Mail On Line, 18 May 2011. 
39. Fear and HOPE, Searchlight Educational Trust, 2011. [http://www.fearandhope.org.uk/projectreport/]

40. “The British workers denied jobs 'because they can't speak Polish'”, London Evening Standard, 19 June 2007 et "Polish workers: Help or Hindrance?", BBC - Kent, 3 April 2008. [http:// www.bbc.co.uk/kent/content/articles/2006/12/11/polish working_feature.shtml].

41. "What does 'British jobs' pledge mean?", BBC NEWS UK, 16 November 2007. [http:// news.bbc.co.uk/2/hi/uk_news/politics/7097837.stm].

42. Roger HEWITT, White Backlash and the Politics of Multiculturalism, New York: Cambridge University, 2005.

43. Arun KUNDNANI, "'Stumbling On": Race, Class and England', Race and Class, 41(4), pp. 1-18.

44. Ibid., p. 12.

45. James RHODES, White Backlash, 'Unfairness' and Justifications of British National Party (BNP) Support, Ethnicities, Vol. 10(1), 2010, pp. 77-99.

46. "Howard: I've had enough of political correctness", Mail On Line, 10 August 2004.[http:// www.dailymail.co.uk/news/article-313489/Howard-Ive-political correctness.html].

47. “Treat anti-white attacks equally', urges Oldham MP”, The Guardian, 3 February 2003. [http:// www.guardian.co.uk/politics/2003/feb/03/immigrationpolicy.equality].

48. “'White voters are deserting us for BNP', says Blair ally", The Telegraph, 16 April 2006. [http:// www.telegraph.co.uk/news/uknews/1515854/White-voters-are-deserting-us-for-BNP-says-Blairally.html].

49. "'White working class feels ignored over immigration', says Hazel Blears", The Guardian, 2 January 2009. [http://www.guardian.co.uk/politics/2009/jan/02/immigration-working-class].

50. Steve GARNER, James COWLES, Barbara LUNG \& Marina SCOTT, Sources of resentment, and perceptions of ethnic minorities among poor white people in England, Report compiled for the National Community Forum, DCLG, London: HMSO, January 2009, pp. 9-10.

51. “'White working class feels ignored over immigration', says Hazel Blears", op.cit.

52. "Theresa MAY: immigration cap will not harm UK economy", The Guardian, 28 June 2010. [ http://www.guardian.co.uk/uk/2010/jun/28/theresa-may-immigration-cap-economy].

53. "English Language Requirement for Spouses", Impact Assessment HO 006, UK Border Agency, London, 1 October 2010.

54. Ibid.

55. "Immigration cap loophole sees massive surge", The Telegraph, 27 November 2010. [http:// www.telegraph.co.uk/news/uknews/immigration/8164911/Immigration-cap-loophole-seesmassive-surge.html].

56. "Immigrants who fail to integrate have created 'discomfort', says Cameron", The Guardian, 14 April 2011. [http://www.guardian.co.uk/politics/2011/apr/14/immigrants-fail-integratediscomfort-cameron?intcmp=239].

57. Ibid.

58. "Vince Cable attacks Cameron's 'very unwise' immigration remarks", The Guardian, 14 April 2011. [http://www.guardian.co.uk/politics/2011/apr/14/vince-cable-david-cameronimmigration-extremism].

59. Net migration to the UK jumps by nearly 100,000, The Guardian, 26 May 2011. [http:// www.guardian.co.uk/uk/2011/may /26/net-migration-uk-jumps-100000].

60. House of Commons, Débat parlementaire du 18 Novembre 2010. [ http:// www.publications.parliament.uk/pa/cm201011/cmhansrd/cm101118/

debtext/101118-0002.htm\#10111862000001].

61. George CAREY, "Migration threatens the DNA of our Nation", The Times, 7 January 2010. [ http://www.timesonline.co.uk/tol/comment/columnists/guestcontributors/article6978389.ece] 62. "David CAMERON : No one will be left behind in a Tory Britain", The Observer, 28 January 2007. [http://www.guardian.co.uk/commentisfree/2007/jan/28/comment.conservatives]. 
63. PM's speech at Munich Security Conference, op.cit.

64. Ibid.

65. Home Office, The Equality Strategy - Building a Fairer Britain, London: HMG, December 2010, p. 24 .

66. Ibid., p. 18.

67. Ibid, p. 7 et "section 5 : Making it happen", pp. 23-24.

68. "Cameron: UK should follow US example on national identity", The Guardian, 5 June 2007. [ http://www.guardian.co.uk/politics/2007].

69. Andrew MYCOCK, Cameron's approach to 'British values' is outdated and divisive, 9 February 2011. [http://www.opendemocracy.net].

70. "David Cameron challenges radical Islamists to a contest of ideas", The Economist, 10 February 2011. [http://www.economist.com/node/18112127].

71. Theresa MAY, 'Our response to the terrorist threat'. This speech was given by the Home Secretary to the Royal United Services Institute (RUSI) on 3 November 2010. [http:// www.homeoffice.gov.uk/media-centre/speeches/terrorist-response].

72. "Quilliam welcomes new UK direction on extremism", Quilliam Foundation, 5 February 2011. [ http://www.quilliamfoundation.org/component/content/article/61-press-releases/761quilliam-welcomes-new-uk-direction-on-extremism.html].

73. "Muslims4Uk says Cameron speech is 'patronising"', BBC News, 5 February 2010. [http:// www.bbc.co.uk/news/uk-politics-12372632].

74. David Cameron and Muscular Liberals, Emle Magazine, 7/8 March 2011. [http://emel.com/ article.php? id=83\&a_id=2300\&c=73\&return=cameron].

75. Liz Fekete citée par Ayesha KAZMI, "It is not multiculturalism that has failed, it is David Cameron", Cageprisoners, 3 March 2011. [http://www.cageprisoners.com/our-work/opinioneditorial/item/1271-it-is-not-multiculturism-that-has-failed-it-is-david-cameron?

tmpl=component\&print=1].

76. "David Cameron : No one will be left behind in a Tory Britain", op.cit.

77. "Cameron 'crusade' to emancipate Muslim women", Mail On Line, 29 January 2007. [http:// www.dailymail.co.uk/news/article-432016/Cameron-crusade-emancipate-Muslim-women.html].

\section{RÉSUMÉS}

Il existe une filiation évidente entre la politique développée par les gouvernements New Labour en matière d'intégration des minorités et de contrôle de l'immigration à partir de 2001 et celle désormais impulsée par le gouvernement de coalition de David Cameron : en particulier, le rejet de la doctrine multiculturaliste et le retour à une vision plus classique de l'intégration. Cependant, il est possible de dégager plusieurs inflexions spécifiques. En premier lieu, une remise en cause extrêmement radicale de l'ensemble de la "politique identitaire " antérieure et son remplacement par une nouvelle "stratégie pour l'égalité ». Ensuite, la volonté gouvernementale d'imposer le système de valeurs britannique aux nouveaux arrivants afin de rétablir un minimum d'homogénéité culturelle, ce qui marque le renouveau de la conception assimilationniste tombée en désuétude au milieu des années 1960. Enfin, la stigmatisation continue et appuyée de l'Islam et des musulmans est devenue une pratique inquiétante qui accentue le «clivage sociétal " que David Cameron dénonçait si fermement lorsqu'il était dans l'opposition. 
There is an obvious link between the policies developed by the New Labour governments as regards ethnic minorities' integration and immigration control after 2001 and the new set of policies recently introduced by David Cameron's coalition government: in particular, the rejection of multiculturalism and the return to a more traditional view of integration. However, several specific trends can be underlined. First, the previous policies based on "identity politics" have been radically challenged and replaced by a new "strategy for equality". Second, the government wants to force the British system of values on the newcomers in order to restore a minimum of cultural homogeneity, which marks the revival of the concept of assimilation abandoned in the mid-1960s. Finally, the continuous and severe stigmatization of Islam and of Muslims has become a worrying tendency which increases the 'community divide' that David Cameron adamantly denounced when he was the leader of the opposition.

\section{AUTEUR \\ DIDIER LASSALLE}

Didier Lassalle est professeur de civilisation britannique à l'Université de Paris Est-Créteil (UPEC). Ses travaux ont essentiellement porté sur l'intégration des minorités ethniques ainsi que sur l'évolution des concepts de citoyenneté et d'identité nationale en Grande-Bretagne. Depuis quelques années, sa recherche a pris une dimension plus résolument comparatiste francobritannique. Il est l'auteur, dans ce domaine, de nombreux articles et de plusieurs ouvrages individuels et collectifs, dont Les Relations interethniques dans l'aire anglophone : entre collaboration(s) et rejet(s), l'Harmattan, 2009, en collaboration avec Lucienne Germain. 